\title{
Ontological Map of Service Oriented Architecture Based on
}

\author{
Zachman \\ Nesa Shafighi ${ }^{1} \&$ Babak Shirazi ${ }^{2 *}$ \\ 1 Department of Information Technology Engineering, Mazandaran University of Science and \\ Technology, Babol, Iran \\ ${ }^{2}$ Department of Industrial Engineering, Mazandaran University of Science and Technology, Babol, Iran \\ *Babak Shirazi, E-mail: shirazi_b@yahoo.com
}

Received: June 16, 2017

Accepted: July 8, 2017

Online Published: July 13, 2017

doi:10.22158/rem.v2n4p33

URL: http://dx.doi.org/10.22158/rem.v2n4p33

\begin{abstract}
Service orientation is an approach in the field of enterprise architecture, business information systems and software application that its main element is the service. Shared services is an organization model of sharing, across an organization. It enables collaboration among the functions/departments. Main motivations for shared services are sharing, promote efficiency, reduce cost, and support scalability. Despite of the widespread use of these two approaches in information technology, there is no tool to optimize the management of them. The aim of this study is Ontological map of service oriented architecture based on zachman framework to adapt it in the reference enterprise architecture framework through implementation ontology views on system architect software and as well as equivalent ontology component with UML diagrams. After the implementation of the suggested model, the results showed that ontology is a formal description and explicit display of objects, concepts and other entities in the relationship between them. In other words, there is a model that describe all that is in fact in to understandable language for the system. Thus the proposed establishes have association between all aspects of zachman framework, also to create a clear description of business concepts in the management of shared services and is effective to provide a unified platform for enterprise modeling.
\end{abstract}

\section{Keywords}

service oriented, shared service, service management, ontology, zachman

\section{Introduction}

Service-oriented architecture is Strategic framework of technology that offers all inside and outside systems to give or receive a well-defined service (Linthicum, 2004). In fact, it is a standard framework that services built, deploy and run in it. Moreover, aims to increase the agility of the information 
technology infrastructure to respond quickly to changes in business needs (Knorr \& Rist, 2005). Service-oriented architecture is an approach to design and implementation organization software by the means of communication between services that have the properties of loose coupling, coarse grains and are reusable (SOA Adoption Blueprint, 2006). In general, service-oriented architecture is an approach to organize and optimize distribution capabilities that territory under the control of the several owner and provides way to order, identify, use and interact with capabilities (Reference Model for Service Oriented Architecture1.0, 2006). Service Oriented Architecture (SOA) and Shared Services (SS) are two correlated streams of research into the alliance of business and information technology (Jeong, Cho, \& Lee, 2009; Papazoglou \& Georgakopoulos, 2003). SOA deals with information architectures that support service oriented computing for on-demand business applications (Stal, 2002; Huhns \& Singh, 2005; Keen et al., 2004). SS, on the other hand, is the consolidation of common functions across multiple organizations to reduce information process duplication and increase information and knowledge sharing (Godse \& Manish, 2012). SS provide new impetus of business sourcing strategies in using information technology. Generally, accounting and financial management, human resources management, acquisition transactions, and customer relation management are the designated lines of business processes for SS (Wang \& Wang, 2007; Rumbaugh et al., 1999; Janssen \& Joha, 2006). Clearly, the major motivation for organizations to adopt SOA is to implement SS (Yuan \& Lu, 2009), and the major technology foundation for the implementation of SS is SOA (Allen, 2006; Jung, 2011). In spite of the close connection between SOA and SS, the two areas are distinct from each other in many aspects. Generally, research reports on SOA have been focusing more on computational technologies (e.g., cloud computing, virtual Web service networks, and software standards), while studies on SS have placed more emphasis on organizational business process management through applications of information technology. From the standpoint of information technology management, integration of the two subjects is imperatively needed to establish SS Management (SSM) that supports the transformation of business process requirements into service-centric computing. In our definition, SSM refers to organizational activities that coordinate the efforts of shared services partners to accomplish desired goals and objectives using shared services efficiently and effectively. The information technology community has called for establishing multidisciplinary service science. One of the approaches that lead to a common understanding of the concepts and elements is enterprise ontology. Ontology comparing with structured, object-oriented and model driven, involve the explicit description of visual elements, components, and various concepts. Also provides integrate semantic context of existing concepts in the business domain for the users. An enterprise architecture framework, defines and organizes all kinds of information in a logical structure. The framework is a tool that helps us to think structural. According to the definitions and studies we find that researches that has been done in the field of service-oriented architecture often deals the identification and application of this type of architecture and models. In addition, the management of shared services with shared resources, requires comprehensive and specific work. On the other hand, studies in the field of enterprise ontology 
often consider the fundamental issues and standards. Although there are gaps in the above-mentioned views that not able to cover interactions between humans and computers in service management properly and provide a way for regular users. In this article, we have tried to present a different mapping than previous methods such as object-oriented model driven by combining two service-oriented and ontology so to recognize enterprise ontology position beyond a concept and push to effective mapping. In addition, mapping that links semantic perspective between all aspects and better management of shared services in the form of a systematic framework since the subject of this study, is a combination of related issues to service-oriented architecture, shared services and ontology mapping. The author has not identified similar research that focused simultaneously on these three elements. The innovation of this study is to combine all three concepts of SOA, SSM and ontology that identify in the form of ZF to take Service Management. This study has six sections the first section includes an introduction, problem definition and the necessity of research is fully described above. The second section is devoted to the literature, concepts of service-oriented architecture, ZF, shared services and ontology are considered. Third section is about history of available techniques and previous works are described. The fourth section describes the proposed model of ontology mapping and we'll explain the method. In the fifth section presents a case study and the last section research result will be discussed.

\section{The Literature}

\subsection{Enterprise Architecture}

An organization has a set of different and distributed tasks. The organization includes numerous resources such as human resources, technology and related structure. Zachman, has been defined enterprise architecture as follow (Zachman \& Sowa, 1992): Enterprise Architecture Provides models in connection with the description of an organization so that conform to the requirements of management, produced and during its life is unmaintainable and changeable.

\subsection{Service-Oriented Architecture}

Service-Oriented Architecture (SOA) is an approach used to create an architecture based upon the use of services. Services (such as Web services) carry out some small function, such as producing data, validating a customer, or providing simple analytical services.

\subsection{Shared Service}

Shared Services is a way of organizing administrative functions to optimize the delivery of cost-effective, flexible, reliable services to all "customers". Shared services is the provision of a service by one part of an organization or group where that service had previously been found in more than one part of the organization or group (Scully \& Levin, 2010; Bergeron, 2003). Thus the funding and resourcing of the service is shared and the providing department effectively becomes an internal service provider. 


\subsection{Ontology}

The word ontology is rooted in metaphysics and philosophical science that is used to describe the nature of being or existence. In general, ontology is defined as a clear description of the common mentality (Ayyazi, 2006). An ontology in a specific domain includes a vocabulary of concepts and conceptual structure that defines the relationship between them. Ontology, in the field of information technology, defined in two ways:

- The ontology defines the terms and concepts by which we can describe the area of knowledge and displayed (Daconta et al., 2003).

- Ontology is a mechanical engineering products, including specific words used to describe the reality and set of explicit hypotheses about the meanings of words (Guarino, 1998).

\subsubsection{Components}

Common components of ontologies include:

Individuals-Instances or objects (the basic or "ground level" objects).

Classes-Sets, collections, concepts, classes in programming, types of objects, or kinds of things.

Attributes-Aspects, properties, features, characteristics, or parameters that objects (and classes) can have.

Relations-Ways in which classes and individuals can be related to one another.

Function terms-Complex structures formed from certain relations that can be used in place of an individual term in a statement.

Restrictions-Formally stated descriptions of what must be true in order for some assertion to be accepted as input.

Rules-Statements in the form of an if-then (antecedent-consequent) sentence that describe the logical inferences that can be drawn from an assertion in a particular form.

Axioms-Assertions (including rules) in a logical form that together comprise the overall theory that the ontology describes in its domain of application. This definition differs from that of "axioms" in generative grammar and formal logic. In those disciplines, axioms include only statements asserted as a priori knowledge. As used here, "axioms" also include the theory derived from axiomatic statements.

Events-The changing of attributes or relations.

\section{Background}

Table 1. Summarizes the Ontology-Based Approaches That Have Been Done

\begin{tabular}{llll}
\hline Year & Journal & Approach & \multicolumn{2}{l}{ Comments } \\
\hline 2005 & Allemang, D., Hodgson, R., \& & Allemang et al., provide & This model is no more than describe \\
& Polikoff, I., Federal Enterprise & FEA-RMO model to a common the framework for enterprise \\
& Architecture reference model & semantic understanding. & architecture FEAF with Web \\
\hline
\end{tabular}


ontologies: FEARMO version

1,1 .

2008

Fuchs-Kittowski, F., \& Faust, D., "The Semantic Architecture Tool (SemAT) for Collaborative Enterprise Architecture

Development", Springer-Verlag Berlin Heidelberg (Vol. 5411, No. 2008, pp. 151-163).

Kang, D., Lee, J., \& Choi, S., An ontology-based Enterprise Architecture, Expert Systems with Applications (pp. 1456-1466).

\begin{abstract}
Ghani, I., Lee, C. Y., \& Juhn S.
\end{abstract}
H.,

"Semantics-oriented approach for

information interoperability

and

governance: towards

user-centric

enterprise

architecture

management",

Zhejiang University-SCIENCE

C (Computers \& Electronics)

(pp. 227-240).
Ontology Language (OWL) and used only for feaf framework.

This model, not provided a clear approach to share meaningful way to the development of architecture and architectural components.

To support the people who are have suggested a architectural involved in the process architecture tool for cooperation in the Collaborative environment is management and development designed like wiki So people and conceptual architecture. groups who are different and have different spaces record information.

Kang et al., presented In the first level business terms, in three-level solution architecture the second level architectural based on ontology to solve the elements and third level relationship lack of a common semantic of the elements considered. Emphasis understanding between different on the use of SBRV approach in systems and between humans enterprise architecture ontologies. and systems As well as between the various stakeholders in an organization that have different semantic understanding about problems.

Ghani et al., have proposed a user-oriented management

Pay attention to users as architecture architecture concept.

Audience. Trying to provide meaningful information for enterprise users that their function is in line with the needs and scope of them.

All of the research are trying to use ontology in architecture, but none of them did not provide a fundamental solution for architecture and didn't pay attention to the enterprise Ontology as 
infrastructure architecture. Also, the process for developing architecture based on ontology are not provided. Zachman framework is a kind of enterprise architecture models are considered Mendeleev table, frame of reference that six of data, processes, places, people, events and objectives under the covers. One of the challenges facing the ZF, the lack of a uniform notation language to cover all aspects and its perspectives. The necessity of such a symbol is that modeling is required between vision and framework as well as ways to communicate and the other using a variety of symbols and language modeling to cover the cells causes confusion and disharmony and makes the architects work difficult and complicated. Following we have mentioned the model used in the development of enterprise architecture based on ontology.

\subsection{The Proposed Model of Enterprise Ontology}

TOVE (Toronto Virtual Enterprise) Project is one of the leading projects in the field of Enterprise ontology creation and has produced A subset of names (Fox Mark et al., 1998) Organization, Resource (Fadel et al., 1994), Activity (Gruninger \& Fox Mark, 1994). For example, the basic concepts underlying the Organization in some columns distributed Zachman. Activity and Resource subsets, respectively, put in the "how" and "who" column. Nevertheless, some columns such as (what) and (Where) is missing (Table 2).

Table 2. Examine the Versatility TOVE Concepts with ZF

\begin{tabular}{lllrlll}
\hline Why & When & Who & & Where & How & What \\
\hline Organization & Time (sub & Organization & Division & ------- & Activity Constraint & \\
goal Sub goal & ontology) & Subdivision & Team & & Resource & \\
Organization & & Organization agent $\quad$ Role & & Communication link & -------- \\
ontology & & Skill Authority & Resource & & Activity & (sub \\
& & & & & \\
& & & ontology) & \\
\hline
\end{tabular}

The second project is The Enterprise Ontology (Uschold et al., 1995). Business organizations gathered in this project is a series of reforms. The main concepts in the proposed project is divided into 5 sections, which are: activities, organization and strategy, marketing and time. For example, concepts related to the activities in the field of "how" and "who" Zachman scattered but for the aspect of "Where" concept cannot be found. So concepts don't cover all aspects of ZF (Table 3).

Table 3. Examine the Versatility the Enterprise Ontology Concepts with ZF

\begin{tabular}{|c|c|c|c|c|c|c|}
\hline Why & When & Who & Where & How & & What \\
\hline Purpose & Time & Person & & Activity & & Entity Role \\
\hline Critical Success & Time Interval & Organization & & Specification & & Relationship \\
\hline Factor & T-Begin T-End & Actor & ---------- & Execute & Plan & Attribute \\
\hline
\end{tabular}




\begin{tabular}{lllll}
\hline Objective & Time & Line & Machine Skill & Process \\
Vision Mission & Calendar Date & Capability & Specification \\
Goal Decision & Duration & Authority & \\
Strategy & & Resource & \\
\hline
\end{tabular}

In the Context-Based Enterprise Ontology (Leppänen, 2007), ontology organization is considered as background. The main objective of the organization is to provide uniform visibility and Total fields. Also considered general concepts for each of the areas. The proposed concept is divided in 7 areas. The target area includes all the concepts that purpose, motivation, intent on anyone and anything refers. Actors include concepts that the human domain, groups, organizational positions and sectors refers active in the field. Field of operation include concepts that the work done in the field or events that refers. Domain object, refers to the operation of information objects and objects of material that it is used. Field of facilities, including all concepts, which by its assets, refers to something done. The local Field, including concepts that are part of the space occupied by some people or some things, refers. Field of time, including concepts that refer to aspects of its time in the field. As can be seen in Table 4 Zachman columns overlap with the areas of corporate ontology. Another advantage of this ontology focus on the production field to the activities of the organization.

Table 4. Examine the Versatility Context-Based Enterprise Ontology Concepts with ZF

\begin{tabular}{lllllrl}
\hline Why & When & Who & Where & How & \multicolumn{2}{l}{ What } \\
\hline aim domain & Time domain & Actor domain & Concepts & Domain & concepts & Concepts of \\
concepts & concepts & concepts & location Field & Activities & and & object Field \\
& & & & facilities & & \\
\hline
\end{tabular}

Another study in the field of ontology mapping service-oriented architecture with the goal of full coverage to ZF (Wang, H., \& Wang, S. H., 2014) the idea is that the relationship between shared services and service-oriented architecture management requirements defined in the sixth aspect creates. In this study, suggest classes that follow from ZF. Including the general meaning has a, is a, implements, locations, involves, activities and associative. Original Perspective ontology shown in Figure 1. 


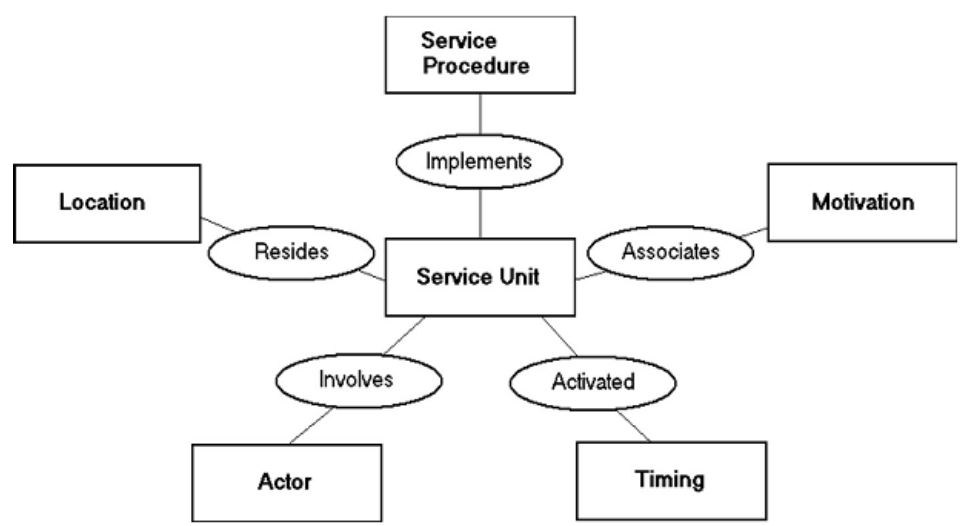

Figure 1. Primary Ontological View of SOA for SSM

This paper proposes an ontological map of SOA for SSM. The Zachman information architecture framework is the foundation of the proposed ontological map. In the history of SOA, modeling SOA has been dominated by software-centered views. On the other hand, the fast growth SS demands the upper-level business-application-centered views. The proposed ontological map provides an instrument to link SOA and SSM. The contribution of this study is the use of the ontology concepts to actualize the six information architecture aspects in the SSM context. In the proposed map, the components of each information architecture aspect are semantically linked into a unified ontology for SSM.

Another model in (Rajabi \& Minaaee bidgoli, 2011) presented the enterprise ontology at the conceptual level that used UML diagram. In this model concepts show as class and the association and aggregation relationships between classes is used to show relationships between concepts of ontology (Figure 2).

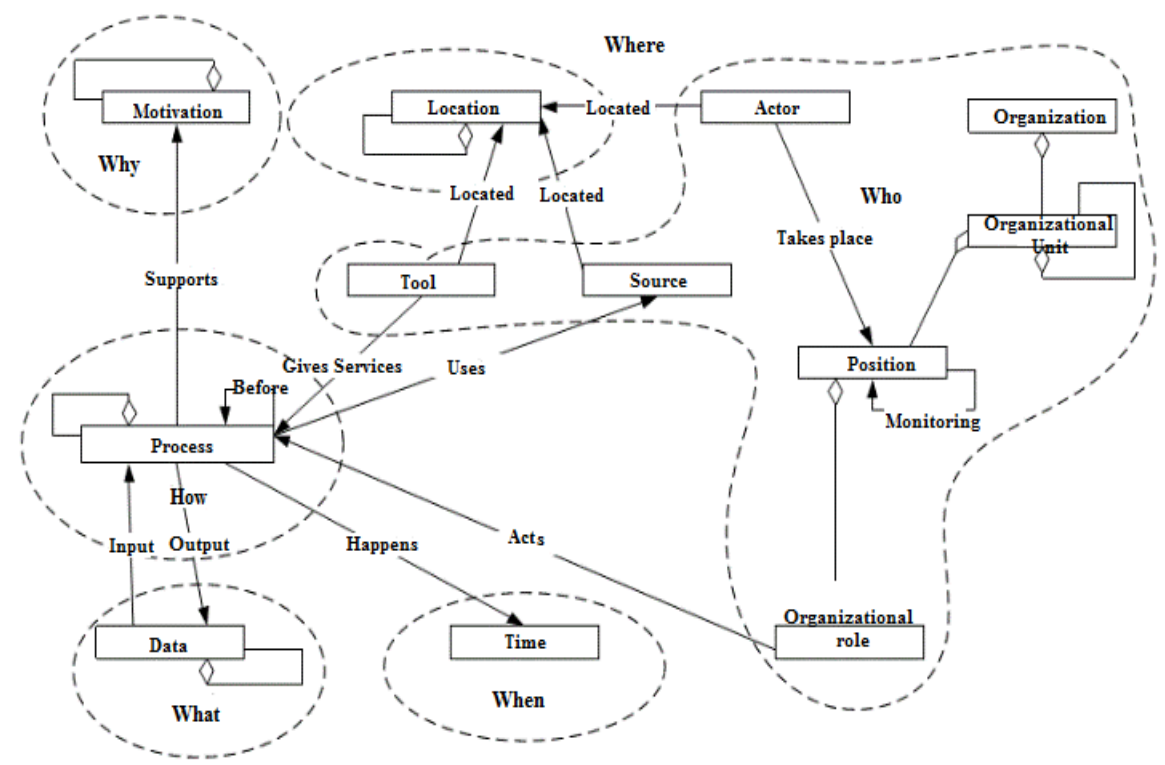

Figure 2. Enterprise Ontology Model Concept-Level and Its Compliance with ZF 


\subsection{Research Gap}

The proposed model presented in is an infrastructure that used in data collection architecture. The proposed concepts so that is intended to be covered aspects of ZF. The versatility of the proposed model of ZF indicates that this model covers all aspects of the organization. But this model is not complete and only show ontology concepts and relationships at the conceptual level. In addition, did not provide a model for other levels. In this article, we have tried to accept the proposed model at the conceptual level (second row) and provide a model for other levels. Note that the first level (Planner) is not a model but a list of things that can be described in natural language. The fifth level (contractor) out-of-themed architecture and its product are not model. In addition, sixth row (the organization is working) is not view but the actual establishment of the components of the organization. Therefore, we are not in the proposed model and is outside the scope of the issue. By accepting the present model on the second level in the next section, we propose a model based on linguistic ontology mapping for third level (designer) and forth level (Producer) of zachman framework.

\section{Ontological Map of Zachman Framework}

One of the applications of ontology is describing the components, concepts and structure of the organization and business. The use of ontologies in the organization, has created a concept called enterprise ontology. Enterprise Ontology is collection of words and definitions related businesses and organizations (Grigoris \& Harmelen, 2008) and usually presented in the form of a classification of areas and concepts scope. Ontology components contains classes, instances, properties, relationships, events, functions, constraints, rules and axioms. Ontological model to cover aspects of ZF components in following will be explained. Since the purpose of the research is providing the ontological model for shared services based on the Zachman Framework. Therefore, to achieve the desired model, Zachman Framework is the base model and diagrams used in the basic Zachman are equivalent with ontology tools and components. In Zachman class and components diagrams were used for data column. We must consider that in the ontology which components do something similar with the diagrams. Since the class is the object-oriented framework for classifying objects there is also the class concept in ontology that categories concepts and entities. So class is used for the categories of entities with common characteristics. In the process column used state diagrams, activity diagrams, sequence diagrams and use-case diagrams to show how implementation processing of activities over time, changing the mode of an object and the interaction between the user and the system. There is also the concept of ontology that describes the relationship between class members and instances that is called relation. Relation described any links and interactive semantic relation between the classes and attributes. Location column contains the geographical distribution of valuable resources for organizations and actor column also includes all those involved and interested in the organization that is used to classify each of the classes. In the Zachman used timing diagrams to described Time column. To display time in the ontology also use the events. Because it indicates the changing scenarios of 
events and relationships and attributes. At the point of time also is a good choice for covering columns are at ZF. There is no definition for motivations Column in the Zachman. In ontology concept of axioms also included rules that form a theory which ontology describes a domain application. So to describe the goals and motivations column this is a good choice. Table 4 shows conversion object-oriented diagrams to the ontology components and tools. After equivalent object-oriented tools to ontology components, model to cover aspects of ZF for each column is explained below.

Table 5. Mapping UML Diagrams to Ontology Component

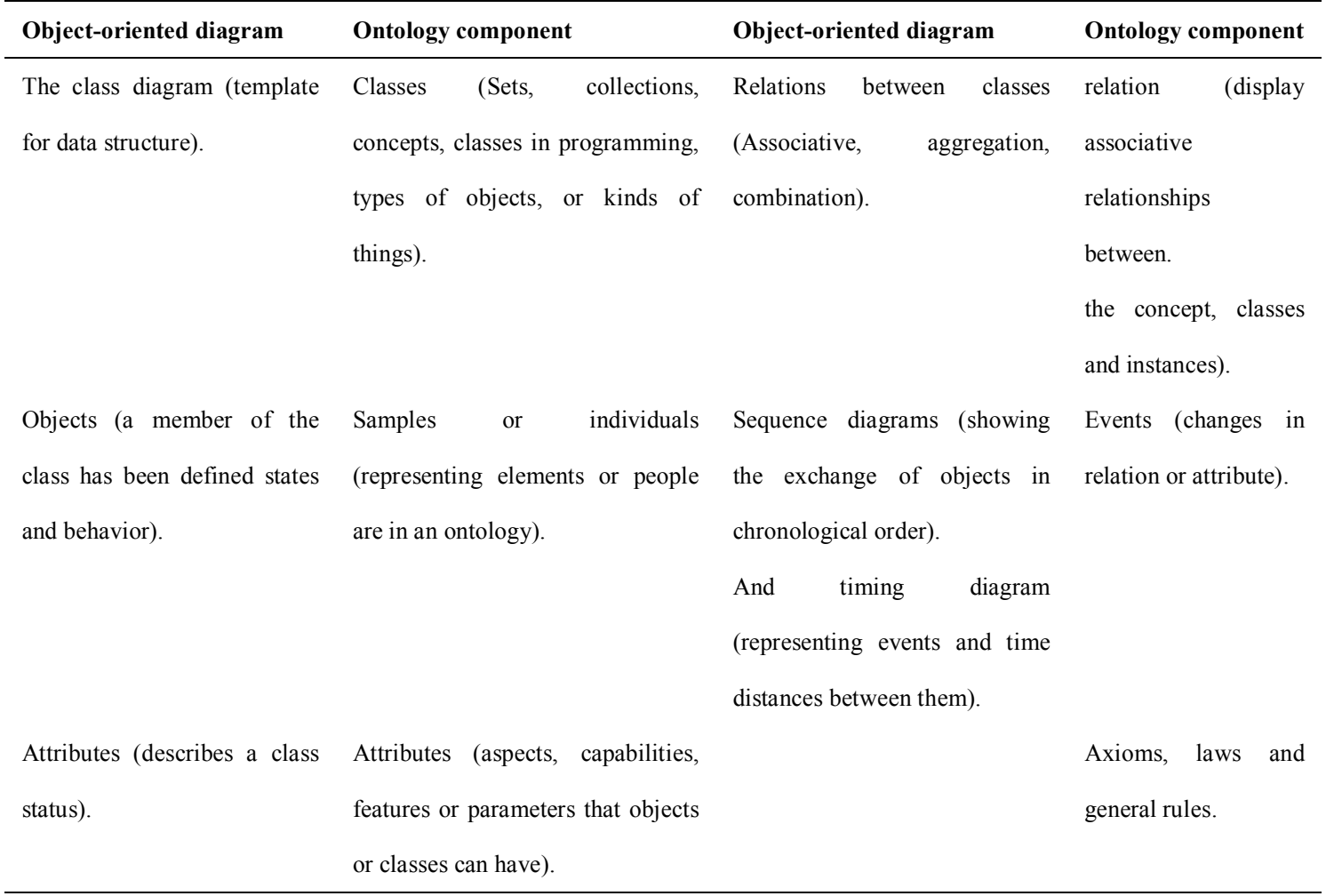

\section{Comparing Model with ZF}

\subsection{Data}

Data is important in the organization and service-oriented architecture and used in the operating cycle. So organizations is useless if there is no data and services to provide there. Instances are the main components of ontology and a member of the class and can include objects, people, tables, animals, plants, molecules, automobiles and, etc. Classes are also groups of entities or objects that have similar characteristics. The classes can be used to describe the "data" used in architecture. In addition, the relationships between data are described by relations between classes. Classes can be hierarchically classified into subclasses with inheritance. So for the display of data in ontology and in answer to the question "what?" can use classes and instances. 


\subsection{Process}

In ontology, relations show kind of associative relationships between the concepts of domain and include classes and examples of ways that they can communicate with each other. Specifications are entities that can exist without specific classes. Relations are connecting class instances to each other. So the proposed method to illustrate how the processes and service process work and in answer to the question "how?" we use relations and attributes in ontology.

\subsection{Location}

Locations refers concepts that are part of the space occupied by some people or some things. Resources and assets of organization are related to this aspect of the ZF. From the ontology perspective to describe the column locations (network) can be used classes that accommodate resource themselves. As well as instances of classes that include organization assets and stock.

\subsection{Time}

Aspect of time is included in ZF to answer the question "When?". Changes occur at the point of time. In ontology to show changes in relationships or attributes used events. Events show switching to another mode by using specific circumstances. So the concept of the event can be used to display time column

\subsection{People}

Those involved in the activities of the organization and includes concepts that refers humans, groups, organizational positions and sectors active in the field. To cover this column we used classes with actor or worker stereotype and instances that are part of the class. In addition use operations part of class, to show the relationship and interaction between people.

\subsection{Motivation}

This column includes, goals, vision, mission, parameters, constraints, success factors and strategies of the organization. To cover this aspect of ZF can be use functions, Constraints (officially Explanations expressed to determine what must be valid until a decision is accepted as input), Rules (sentences express if then, inference) and axiom (including rules) in ontology. Table 6 shows ontology model for covering the third and fourth rows of ZF.

Table 6. Ontological Model for the Cover of ZF

\begin{tabular}{lllllllll}
\hline Why & When & Who & Where & & How & What \\
\hline Functions Rules & Event & Class (with actor and worker & Class $\quad($ with & client & and & Relations & Class & (with \\
Constraints Axiom & & stereotype) Instance & server stereotype) Instance & Attributes & stereotype) Instance
\end{tabular}

\section{Conclusion}

In this paper we have tried to provide a different mapping than previous methods such as object-oriented and Model driven architecture approach, by combining with service-oriented and 
ontology to recognize Enterprise ontology position beyond its concept. This is achieved by implementing and adapting ontology vision with Enterprise Architecture Framework. Another purpose is providing the semantic mapping between all aspects and based on this, the proposed model covers all aspects. Considering that service management contains a set of Specific organizational capabilities for providing value to customers in the form of service. The proposed ontology mapping enables managers to think organized in a systematic and reference framework form in the field of enterprise architecture and make decision based on the model that covers all aspects of service requirements.

\section{References}

Allen, P. (2006). Service orientation: Wining strategies and best practices. Cambridge, UK: Cambridge University Press. https://doi.org/10.1017/CBO9780511541186

Ayyazi, F. (2006). Organization Memory and its role in improving organizational performance, Master's Seminar Report, Islamic Azad University, Science and Research, Faculty of Engineering. Department of Computer Engineering and Robotics.

Bergeron, B. (2003). Essential of shared services. John Wiley \& Sons.

Daconta, M. C., Smith, K. T., \& Obrst, L. J. (2003). The Semantic Web: A Guide to the Future of XML, Web Services, and Knowledge Management. John Wiley \& Sons, Inc., New York NY, USA.

Fadel, F. G., Fox, M. S., \& Gruninger, M. (1994). A Generic Enterprise Resource Ontology, USA. In Proceedings of the 3rd Workshop on Enabling Technologies: Infrastructure for Collaborative Enterprise (pp. 117-128). https://doi.org/10.1109/ENABL.1994.330496

Fox, M. S., Barbuceanu, M., Gruninger, M., \& Lin, J. (1998). An Organization Ontology for Enterprise Modelling. In Simulating Organizations: Computational Models of Institutions and Groups (pp. 131-151).

Godse, M. (2012). Exploiting the Values of Shared Services. Third International Conference on Services in Emerging Markets. https://doi.org/10.1109/ICSEM.2012.31

Grigoris, A., \& Frank van, H. (2008). A Semantic Web Primer (2nd ed.) (Cooperative Information Systems).

Gruninger, M., \& Fox, M. S. (1994). An Activity Ontology for Enterprise Modelling.

Guarino, N. (1998). Formal Ontology in Information Systems, proceedings of the First International Conference (FOIS'98). Trent, Italy, IOS Press.

Hai, W., \& Wang, S. H. (2014). Ontological map of service oriented architecture for shared services management. Expert Systems with Applications, 41, 2362-2371. https://doi.org/10.1016/j.eswa.2013.09.034

Huhns, M. N., \& Singh, M. P. (2005). Service-oriented computing: Key concepts and principles. IEEE Internet Computing, 9(1), 75-81. https://doi.org/10.1109/MIC.2005.21

Janssen, M., \& Joha, A. (2006). Motives for establishing shared service centers in public administrations. International Journal of Information Management, 26(2), 102-112. 
https://doi.org/10.1016/j.ijinfomgt.2005.11.006

Jeong, B., Cho, H., \& Lee, C. (2009). On the functional quality of service (FQoS) to discover and compose interoperable web services. Expert Systems with Applications, 36(3), 5411-5418. https://doi.org/10.1016/j.eswa.2008.06.087

Jung, J. J. (2011). Service chain-based business alliance formation in service oriented architecture. Expert Systems with Applications, 38(3), 2206-2211. https://doi.org/10.1016/j.eswa.2010.08.008

Keen, M. et al. (2004). Patterns: Implementing an SOA Using an Enterprise Service Bus, IBM Redbooks (July).

Knorr, E., \& Rist, O. (2005). 10 Steps to SOA. Retrieved from http://weblog.infoworld.com/article/05/11/07/45FEsoastep1_1.html

Leppänen, M. (20047). A Context-Based Enterprise Ontology (pp. 273-286). Springer Berlin/Heidelberg. https://doi.org/10.1007/978-3-540-72035-5_21

Linthicum, D. (2004). What Level Is Your SOA? Choose for what you need and maybe a little better. Retrieved from http://webservices.sys-con.com/read/47277.htm

Oasis: Reference Model for Service Oriented Architecture1.0. (2006). Retrieved from http://docs.oasis-open.org/soa-rm/v1.0/soa-rm.pdf

Oasis: SOA Adoption Blueprint. (2006). Retrieved from http://www.oasis-open.org/committees/download.php/17616/06-04-00002.000.doc

Papazoglou, M. P., \& Georgakopoulos, D. (2003). Service-oriented computing: Introduction. Communications of the ACM, 46(10), 24-28. https://doi.org/10.1145/944217.944233

Rajabi, Z., \& Minaaee, B. B. (2011). Providing a solution for enterprise architecture based on ontology. First National Conference on Computer and IT students.

Rumbaugh, J., Jacobson, I., \& Booch, G. (1999). The unified modeling language reference manual. Boston, MA: Addison-Wesley Sako, M.

Scully, J., \& Levin, B. (2010). HR shared services is hot and getting hotter. Employment Relations Today. In Wiley Inter-science (pp. 23-30).

Stal, M. (2002). Web services: Beyond component-based computing. Communications of the ACM, 45(10), 71-77. https://doi.org/10.1145/570907.570934

Uschold, M., King, M., Moralee, S., \& Zorgios, Y. (n.d.). The Enterprise Ontology. Artificial Intelligence Application Institute (AIAI), the Univercity of Edinburgh, 1995.

Wang, S., \& Wang, H. (2007). Shared services beyond sourcing the back offices: Organizational design. Human Systems Management, 26(4), 281-290.

Yuan, S. T., \& Lu, M. R. (2009). A value-centric event driven model and architecture: A case study of adaptive complement of SOA for distributed care service delivery. Expert Systems with Applications, 36(2), 3671-3694. https://doi.org/10.1016/j.eswa.2008.02.024

Zachman, J. A., \& Sowa, J. F. (1992). Extending and Formalizing the Framework for Information Systems Architecture. IBM Systems Journal, 31(3). 